\title{
Bioanalysis
}

\section{DBS and beyond}

\author{
"...the articles published in this special edition have demonstrated that \\ our understanding of the limitations of [DBS] technology is increasing and \\ that multiple groups are progressing with approaches to overcome, \\ or minimize these limitations..."
}

\section{Keywords: bioanalysis $\bullet$ DBS $\bullet$ microsampling $\bullet$ quantitative}

\section{Background}

Although matrix microsampling, and specifically DBS sampling, are not new approaches for the convenient collection of blood samples for subsequent analysis, the advent of their more recent use for the quantitative determination of circulating drug concentrations in drug development studies [1], for therapeutic drug monitoring (TDM) [2] and toxicology [3] has led to an upturn in interest and publications (Figure 1). This continued and growing interest in microsampling and DBS sampling demonstrates that laboratories are eager to realize the many benefits of these approaches, which have been well described elsewhere [4].

Currently, the numbers of publications in this field are doubling every 2-3 years, with many of these articles being published in Bioanalysis. At the time of writing, 10 out of the 20 most cited articles in Bioanalysis concern microsampling and DBS technologies [6] and prior to this special issue, 189 articles have been published on this subject in Bioanalysis [5]. Building on the previous special editions of Bioanalysis on this subject in 2010 (Volume 2, Issues 8 and 11) and 2013 (Volume 5, Issue 17), this special edition has been compiled with the aim of examining recent progress in this rapidly developing field and where it might take us in the future.

\section{Scope}

The objective of this special edition of Bioanalysis is to highlight the ongoing developments in microsampling and dried matrix sampling technologies and their routine implementation. Specifically, this issue contains manuscripts covering the following:
- 'Ask the Experts' around the benefits, approaches, drawbacks, implementation and future outlook for microsampling and dried matrix sampling;

- Understanding the effect of drying on DBS samples;

- Novel approaches for dried blood sampling technology to enable the simple collection of accurate blood volumes and novel substrates to minimize the effect of blood hematocrit on blood spot size;

- The exploration of a novel membrane to produce dried plasma spots;

- Automation of DBS sample analysis, with an emphasis on overcoming hematocrit-based biases;

- Implementation of DBS sampling for home sampling, therapeutic drug monitoring, pediatric monitoring and toxicological analysis.

\section{Looking forward}

The continuing increase in the number of publications for DBS technology demonstrates the continued interest in the use and development of this technique and its practical routine implementation. Furthermore, the articles published in this special edition have demonstrated that our understanding of the limitations of the technology is increasing and that multiple groups are progressing with approaches to overcome, or minimize these limitations, particularly those associated with blood hematocrit and sample homogeneity. In addition, we are collectively finding novel

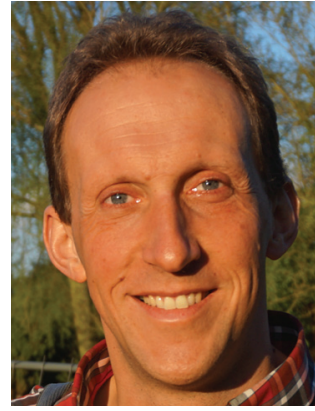

Christophe Stove Laboratory of Toxicology, Faculty of Pharmaceutical Sciences, Ghent University, Ottergemsesteenweg 460, 9000 Ghent, Belgium

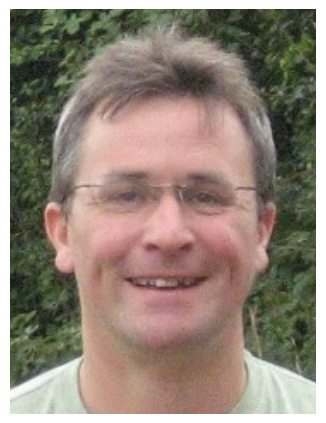

Neil Spooner

Author for correspondence: Bioanalytical Science \& Toxicokinetics, Drug Metabolism \& Pharmacokinetics, GlaxoSmithKline R\&D, Ware, UK neil.spooner@gsk.com 


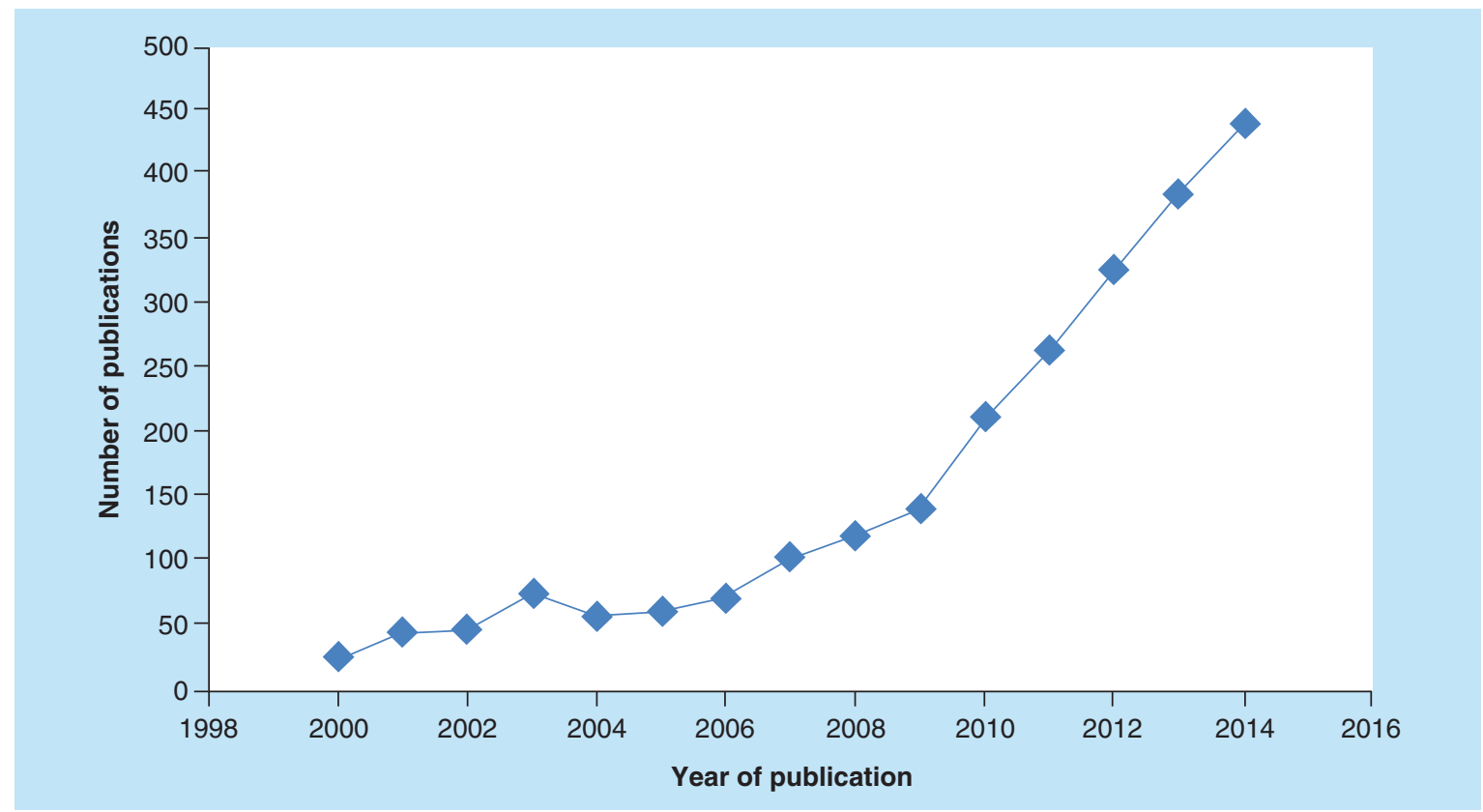

Figure 1. Number of publications per year in the field of DBS since 2000.

Data obtained from [5], using the search term 'dried blood spots' in the title, abstract or keywords.

ways to collect high-quality samples - a prerequisite for obtaining high-quality data. Furthermore, the possibility of automating analysis enhances the ease of generating meaningful data for routine decision making. While the use of DBS sampling in drug development studies may have stalled, many recent publications, including several in this issue, clearly demonstrate that the technology is thriving where used for TDM and home sampling. The authors believe that home-based patient self-sampling may result in a cost-effective public health advantage in various fields and may - in time - become routinely implemented for patient follow-up or diagnostics, as is actually already the case in some clinical centers. The authors hope that the continued implementation of these techniques in these areas will in-time lead to their more ready acceptance for highly regulated clinical drug development studies, thus realizing the benefits of this form of sampling. Obviously, challenges will remain, as with any technology, and microsampling (dried or liquid) may

\section{References}

1 Spooner N, Lad R, Barfield M. Dried blood spots as a sample collection technique for the determination of pharmacokinetics in clinical studies: considerations for the validation of a quantitative bioanalytical method. Anal. Chem. 81, 1557-1563 (2009).

2 Edelbroek PM, van der Heijden J, Stolk LML. Dried blood spot methods in therapeutic drug monitoring: methods, assays and pitfalls. Ther. Drug Monit. 31(3), 327-336 (2009). not be an option for every compound or condition. Studies such as those in this special issue will definitely help to pave the way. A lot remains to be explored and more new developments are underway to make the technique even more accessible and fool-proof. In conclusion, we remain convinced that the pond of dried blood applications would not become dry soon and that there may be a large potential ahead, where in many cases the challenges may be outweighed by the benefits.

\section{Financial \& competing interests disclosure}

The authors have no relevant affiliations or financial involvement with any organization or entity with a financial interest in or financial conflict with the subject matter or materials discussed in the manuscript. This includes employment, consultancies, honoraria, stock ownership or options, expert testimony, grants or patents received or pending, or royalties.

No writing assistance was utilized in the production of this manuscript.

3 Stove CP, Ingels AS, De Kesel PM, Lambert WE. Dried blood spots in toxicology; from the cradle to the grave? Crit. Rev. Toxicol. 42, 230-243 (2012).

4 Spooner N. A glowing future for dried blood spot sampling. Bioanalysis 2(8), 1343-1344 (2010).

5 Scopus search of titles, abstracts and keywords using the term 'dried blood spot'. www.scopus.com

6 Bioanalysis most frequently cited papers. www.future-science.com 